TITLE:

\title{
Fluctuation scaling of quotation activities in the foreign exchange market
}

\section{AUTHOR(S):}

Sato, Aki-Hiro; Nishimura, Maiko; Hoyst, Janusz A.

\section{CITATION:}

Sato, Aki-Hiro ...[et al]. Fluctuation scaling of quotation activities in the foreign exchange market. Physica A: Statistical Mechanics and its Applications 2010, 389(14): 2793-2804

\section{ISSUE DATE:}

2010-07-15

URL:

http://hdl.handle.net/2433/120957

\section{RIGHT:}

(c) 2010 Elsevier B.V.; This is not the published version. Please cite only the published version.; この論文は出版社版でありません。引用の際に は出版社版をご確認ご利用ください。 


\title{
Fluctuation scaling of quotation activities in the foreign exchange market
}

\author{
Aki-Hiro Sato ${ }^{\mathrm{a}, *}$ Maiko Nishimura ${ }^{\mathrm{a}}$ Janusz A. Hołyst ${ }^{\mathrm{b}}$ \\ a Department of Applied Mathematics and Physics, Graduate School of Informatics, \\ Kyoto University, Kyoto 606-8501, Japan \\ ${ }^{\mathrm{b}}$ Faculty of Physics and Center of Excellence for Complex Systems Research, \\ Warsaw University of Technology, Koszykowa 75, PL-00-662 Warsaw, Poland
}

\begin{abstract}
This study investigates scaling behavior of quotation activities in the foreign exchange market. The power-law relationship between a mean of quotation activities and their standard deviation for each currency pair is found, and the dependence of the scaling exponent $\alpha$ on the time window $\Delta t$ is calculated. It is found that the scaling exponent fluctuates temporally in a range from 0.8 to 0.9 at $\Delta t=1$ [min], depending on observation days. The extraction between specific fluctuations and a common fluctuation from quotation activities is conducted. It is concluded that quotation activities in the foreign exchange market are not independently Poissonian, and that temporally or mutually correlated activities of quotations happen. We propose a stochastic model for the foreign exchange market based on a bipartite graph representation. The components' centrality on a bipartite graph is estimated from multiple time series and visualized on a currency pair network. Consequently, the scaling exponents can be used to quantify market participants' states based on information flows in the foreign exchange market. We found that as increasing the window length market participants are affected by exogenous field.
\end{abstract}

Key words:

PACS: 05.40.-a, 02.50.-r, 89.75.Da

Econophysics, The foreign exchange market, power law scaling, Taylorism, quotation activities

\section{Introduction}

Recently many researchers have paid considerable attention to both economically and socially motivated problems in the literature of complex systems [1-8]. Collective be-

\footnotetext{
* corresponding author.

Email address: aki@i.kyoto-u.ac.jp (Aki-Hiro Sato).
} 
havior among interacting agents shows different properties from particles governed by Newtonian laws. However, intriguing properties could be found and mathematical models should be considered. This movement, called socio/econo-physics, is expected to bridge a gap between physics and our societies [9].

Financial markets are complex systems which consist of many interacting agents. The progress of understanding information flows among agents sheds the light on states of financial markets, i.e. states of market participants. The recent accumulation of a massive amount of data about financial markets due to both the development and spread of information and communication technology, allows us to quantify the states of financial markets in detail $[10,11]$. In fact, the correlation structure of high-frequency financial time series are exhaustively and quantitatively investigated [12,13], however, it becomes more difficult to compute cross-correlations and to recognize their numerical results as the dimension of multiple time series increases further.

On the other hand, several pieces of research in both socio/econo-physics and engineering have a remarkable interest in both the structure of complex networks $[14,15]$ and the flows of the constituents on them. Several studies investigate social or artificial constructions from a viewpoint of complex networks. Menezes and Barabási studied the scaling behavior of constituents' flows on several constructions such as river networks (water flows), transportation systems (car flows), and computer networks (information flows) $[16,17]$. As a result, scaling properties are found for flow fluctuations in such systems. This relationship is known as fluctuation scaling or Taylor's power law $[21,22]$. The Taylor's power law is known as the scaling-relationship between the mean of populations and their standard deviation in ecological systems. The ubiquity of Taylor's power-law slopes of between $1 / 2$ and 1 suggests that there exists an underlying fundamental mechanism affecting the transportation of constituents.

Eisler and Kertész reported that there is such a power-law relationship between a mean of traded volumes of stocks and their standard deviation in the New York Stock Exchange, and that the power-law exponent takes a nontrivial value between $1 / 2$ and 1 [23]. Jian et al. investigated trade volumes of stocks in the Chinese stock market [24]. They found that they show a non-universal scaling exponent of fluctuations different from $1 / 2$ and 1 . We think that their results provide a method to quantify the states of agents with multiple time series in the financial markets. This method is also useful to quantify the states of market participants from the viewpoint of information flows in the financial markets.

The aim of this article is to investigate into information flows in the foreign exchange market (FX market) by means of quotation activities, measured as arrival rates of quotations on broking systems. We investigate the fundamental properties of quotation activities in the FX market and quantify the total states of the market participants through fluctuation scaling.

The organization of this article is as follows. In Sec. 2 analyzed high-frequency financial data of the FX market is presented. In Sec. 3 a brief explanation of a power-law relationship between a mean of constituents' flow in complex networks and their standard deviation is shown. In Sec. 4 empirical analysis of quotation activities is conducted. In Sec. 5 the dependence of scaling exponents on a time window is examined, and the relationship between the states of market participants in the FX market and the scaling exponents is discussed. Sec. 6 is devoted to concluding remarks and shows future works. 


\section{Foreign exchange market}

The foreign exchange market is the largest financial market in the world. It is a network consisting of brokers, bank traders, and investors. Recent developments in Information and Communication Technology have lead to the spread of electronic trading systems all over the world. As a result, many market participants can directly access to the FX market by using computer terminals. Moreover, trading activities are recorded in the server computers, which conduct the matching operations among quotations from the market participants, and we can analyze a large amount of data about market activities with a high resolution.

In the analysis we use Time \& Sales (T\&S) data provided by CQG Inc. [25]. The data contains time stamps, rates, and indicators to show ask or bid quotations with a 1 minute resolution. The database includes 45 currency pairs ${ }^{1}$ consisting of 24 currencies.

Quotation activities for each currency pair are extracted from the database. Since the two-way quotation is adopted in the FX market, it is enough to count the number of the bid or ask quotations.

Let $X_{i, \Delta t}(t) \quad(i=1, \ldots, N ; t=0, \Delta t, 2 \Delta t, \ldots,(Q-1) \Delta t)$ denote the number of all incoming ask quotations for a period between $t \Delta t$ and $(t+1) \Delta t$ for the currency pair i. $\Delta t(>0)$ denotes the time window to compute the number of quotations. Examples of quotation activities for EUR/JPY, USD/JPY and EUR/USD are shown in Fig. 1. We shall treat the FX market as a complex system consisting of 45 sites $i$ with unknown number of internal variables, where every site $i$ is a corresponding currency pair from our database and its internal activity is given by the number of quotations $X_{i, \Delta t}(t)$. It is confirmed that the quotation activities exhibit an intraday pattern related to the rotation of the earth, so that they have a strong regional dependence due to entering and leaving of market participants. We focus on short-term behavior of quotation activities under the assumption of local stationarity. In the context of financial markets the number of quotations or transactions is known to represent a proxy variable of the latent number of information arrivals in the system [26], where the term "information" is defined as "a difference which makes a difference" [27].

\section{Theory}

\subsection{Fluctuation Scaling}

In a complex network in which constituents are transmitted between nodes, we focus on the number of constituents which arrive on the node $i$ during $\Delta t$, which is denoted as $X_{i, \Delta t}(t)$. In the context of financial markets the number of quotations or transactions is known to represent a proxy variable of the latent number of information arrivals in

1 The database contains AUD/CHF, AUD/JPY, AUD/NZD, AUD/SGD, AUD/USD, CAD/CHF, $\mathrm{CAD} / \mathrm{JPY}, \mathrm{CHF} / \mathrm{JPY}, \mathrm{EUR} / \mathrm{AUD}, \mathrm{EUR} / \mathrm{CHF}, \mathrm{EUR} / \mathrm{CZK}, \mathrm{EUR} / \mathrm{GBP}$, EUR/HUF, EUR/JPY, EUR/NOK, EUR/PLN, EUR/SEK, EUR/TRL, EUR/USD, GBP/AUD, GBP/CAD, GBP/CHF, GBP/INR, GBP/JPY, GBP/NZD, GBP/USD, NZD/USD, USD/BRL, USD/CAD, USD/CHF, USD/DKK, USD/HKD, USD/HUF, USD/INR, USD/JPY, USD/KRW, USD/MXN, USD/NOK, USD/PKR, USD/PLN, USD/SEK, USD/SGD, USD/THB,USD/TRL, and USD/ZAR. These currency names are described based on ISO 4217 . 
the system [26], where the term "information" is defined as "a difference which makes a difference" [27].

It is known that the mean of $X_{i, \Delta t}(t)$

$$
\left\langle X_{i, \Delta t}\right\rangle=\frac{1}{Q} \sum_{j=0}^{Q-1} X_{i, \Delta t}(j \Delta t)
$$

and its standard deviation

$$
\sigma_{i, \Delta t}=\sqrt{\frac{1}{Q} \sum_{j=0}^{Q-1}\left(X_{i, \Delta t}(j \Delta t)-\left\langle X_{i, \Delta t}\right\rangle\right)^{2}},
$$

follow a power-law relationship

$$
\sigma_{i, \Delta t}=C\left\langle X_{i, \Delta t}\right\rangle^{\alpha}
$$

where $C$ is a positive constant, and $\alpha \quad(1 / 2 \leq \alpha \leq 1)$ denotes a scaling exponent. This power-law relationship is referred to as the fluctuation scaling, or Taylors' law [21,22].

The situations where $\alpha$ takes $1 / 2$ for every time window size are as follows. (1) The first possibility can correspond to standard fluctuations observed in equilibrium statistical physics where dispersion of volume of the total magnetization scales of the size of the system as the power $1 / 2$. (2) The second possibility can be a Poissonian like process with very low activity where one can neglect the square of the mean of activity comparing to the mean activity itself. On the other hand the situations where $\alpha$ takes 1 for every time window size are as follows. (1) One case corresponds to the situation when every node $i$ is driven by universal(common) random variable. $\left\langle V_{i}(t)\right\rangle$ are mean values of $V_{i}(t)$ is the same for every node $i$ but the individual activity of every node is dependent also on some constants $F_{i}$, i.e. $f_{i}(t)=F_{i}\left\langle V_{i}(t)\right\rangle,\left\langle f_{i}\right\rangle=f_{i}\left\langle V_{i}\right\rangle$. (2) The possible scenario can be the case where the activity for every node $i$ is the some of the activities of identical completely synchronized constituents. As another scenario that $\alpha$ takes from $1 / 2$ to 1 , the Hurst exponents' heterogeneity is known [22].

\subsection{Specific fluctuations and common fluctuation}

According to Ref. [17] we can separate specific and common fluctuations of complex systems as follows. This can be based on the assumption that a common fluctuation may be originated from exogenous factors of elements and that specific ones come from endogenous factors. Of course a common fluctuation is not always originated from a common exogenous factor. It is known that synchronization can be happened due to interaction among elements $[18,19]$. We assume that we can separate the two contributions by writing

$$
X_{i, \Delta t}(t)=X_{i, \Delta t}^{\mathrm{spe}}(t)+X_{i, \Delta t}^{\mathrm{com}}(t)
$$

It is proposed that a time independent fraction $A_{i}$ of the total quotation is determined by the component's centrality [20]. We can write $A_{i}$ as the ratio of the total quotations that have arrived on matching engines for the currency pair $i$ in the time interval $t \in[0, Q-1]$, and the total quotations over all observed components during the same time interval

$$
A_{i}=\frac{\sum_{j=0}^{Q-1} X_{i, \Delta t}(j \Delta t)}{\sum_{j=0}^{Q-1} \sum_{i=1}^{N} X_{i, \Delta t}(j \Delta t)} .
$$


Therefore, the common fluctuation that contributes to the activity of the currency pair $i$ is expected to be

$$
X_{i, \Delta t}^{\mathrm{com}}(t)=A_{i} \sum_{i=1}^{N} X_{i, \Delta t}(t) .
$$

The scaling exponent of $X_{i, \Delta t}^{\mathrm{com}}(t)$ always takes 1 from the direct consequence of its definition.

From Eqs. (4) and (6) the specific fluctuations are given by

$$
X_{i, \Delta t}^{\mathrm{spe}}(t)=X_{i, \Delta t}(t)-\left(\frac{\sum_{j=0}^{Q-1} X_{i, \Delta t}(j \Delta t)}{\sum_{j=0}^{Q-1} \sum_{i=1}^{N} X_{i, \Delta t}(j \Delta t)}\right) \sum_{i=1}^{N} X_{i, \Delta t}(t) .
$$

Furthermore, by means of Eqs. (6) and (7), we can determine whether the fluctuations observed in a system are synchronous or asynchronous, so that they may be affected by endogenous or exogenous contributions. From each currency pair we calculate the standard deviations of both specific fluctuations and a common fluctuation,

$$
\begin{aligned}
\sigma_{i, \Delta t}^{\mathrm{spe}} & =\sqrt{\left\langle X_{i, \Delta t}^{\mathrm{spe}}(t)^{2}\right\rangle-\left\langle X_{i, \Delta t}^{\mathrm{spe}}(t)\right\rangle^{2}}, \\
\sigma_{i, \Delta t}^{\mathrm{com}} & =\sqrt{\left\langle X_{i, \Delta t}^{\mathrm{com}}(t)^{2}\right\rangle-\left\langle X_{i, \Delta t}^{\mathrm{com}}(t)\right\rangle^{2}},
\end{aligned}
$$

and their ratio

$$
\eta_{i}=\frac{\sigma_{i, \Delta t}^{\mathrm{com}}}{\sigma_{i, \Delta t}^{\mathrm{spe}}} .
$$

From definition $\left\langle X_{i, \Delta t}^{\operatorname{com}}\right\rangle=0$. If $\eta_{i}>1$, the common fluctuation is dominant in the dynamics of the currency pair $i$. Otherwise, the specific fluctuations are dominant. Moreover, it is known that the common fluctuation scales as a power law with the exponent $\alpha=1$, when we plot a standard deviation $\sigma_{i, \Delta t}^{\mathrm{com}}$ as a function of a mean $\left\langle X_{i, \Delta t}\right\rangle$. The scales of the specific fluctuation provide us with a more detailed understanding of the system's dynamics.

\section{Data Analysis}

For each currency pair, quotation activities are extracted from a T\&S database. We calculate a mean of the quotation activities and their standard deviation for each currency pair following Eq. (1) and (2). As shown in Fig. 2 a power-law relationship between a mean and standard deviation in $\log$-log plots is found. In order to estimate $C$ and $\alpha$ we used the least square method for $\log \left\langle X_{i, \Delta t}\right\rangle$ and $\log \sigma_{i}$. From a double logarithmic form of Eq. (3) the fitting function is set as

$$
\log \sigma_{i, \Delta t}=\alpha \log \left\langle X_{i, \Delta t}\right\rangle+\log C .
$$

Following this procedure, the power-law exponent is estimated as 0.8 at $\Delta t=1$ [min], as 0.85 at $\Delta t=10[\mathrm{~min}]$, and as 0.876 at $\Delta t=120[\mathrm{~min}]$ for a period from the 2 nd to 6 th July, 2007.

Furthermore, the dependences of the $\alpha$ on $\Delta t$ are computed for 4 periods in July 2007. One can see that the exponent $\alpha$ is almost constant for longer time windows, $\Delta t>100$ [min], see Fig. 3 (top). On the other hand for shorter time scales this exponent 
increases proportionally to $\log \Delta t$ up to $\Delta t=10$ [min]. Slopes of this increase are almost independent of observation days.

As shown in Fig. 4, the $\alpha$ value varies temporally in a range from 0.8 to 0.9 at $\Delta t=1$ [min]. This temporal variation can be related to the temporal dependence of the synchronous and desynchronous activities of the quotation for each currency pair arriving on brokerage systems on trading days. Furthermore as $\Delta t$ increases the common fluctuation has a tendency to become dominant. This result is consistent with the dependences of $\alpha$ on $\Delta t$, as shown in Fig. 3.

Following Eq. (5) we calculated the centrality of each currency pair for $T=1440$ [min] and drew the currency pair network with links corresponding to its centrality as shown in Fig. 5. From Fig. 5 we can find that USD/CHF, AUD/JPY, CHF/JPY, EUR/AUD, EUR/GBP, EUR/JPY, EUR/USD, GBP/AUD, GBP/CAD, GBP/CHF, GBP/JPY, GBP/USD, USD/CHF, USD/DKK, USD/NOK, USD/SEK have a large value as its centrality.

As shown in Fig. $6 \eta_{i}$ are distributed dependently on the time window. As $\Delta t$ increases, the distribution shift from the negative side of $\log \eta_{i}$ to its positive side. Tab. 1 shows the values of $\log \eta_{i}$ for each $\Delta t$. From this table we find that some currency pairs are dominated by the specific fluctuations, and that others by the common fluctuation. The currency pairs in which the specific fluctuations are dominant are EUR/CZK, GBP/INR, USD/BRL, USD/HKD, USD/INR, USD/KRW, USD/MXN, USD/PKR, USD/SGD, USD/THB, USD/TRL, and USD/ZAR. The currency pairs in which a common fluctuation is dominant are USD/CHF, AUD/JPY, CAD/CHF, CHF/JPY, EUR/AUD, EUR/GBP, EUR/JPY, EUR/USD, GBP/AUD, GBP/CAD, GBP/CHF, GBP/JPY, GBP/NZD, GBP/USD, USD/CHF, USD/DKK, USD/HUF, USD/JPY, USD/PLN, and USD/SEK. Consequently, rare currency pairs tend to be subject to the specific fluctuations, while hard currency pairs tend to be subject to the common fluctuation. It is confirmed that quotations of currency pairs with a high centrality commonly fluctuate from Figs. 5 and 6.

Moreover the relationship between $\alpha$ and $\Delta t$ shown in Fig. 3 is consistent with the results that the mean value of $\eta_{i}$ moves to a positive direction as $\Delta t$ increases (See. Fig. $6)$.

By plotting for each currency pair, the standard deviations $\sigma_{i}^{\text {com }}$ and $\sigma_{i}^{\text {spe }}$ as a function of the average $\left\langle X_{i, \Delta t}\right\rangle$, we can understand the dynamics of these fluctuations. As shown in Fig. 7 the common fluctuations scale with $\alpha=1$. By using Eq. (11) it is found that the specific fluctuation scales with $\alpha=0.687$ at $\Delta t=1[\mathrm{~min}], \alpha=0.684$ at $\Delta t=10$ [min], and $\alpha=0.656$ at $\Delta t=120[\mathrm{~min}]$. Hence, $X_{i, \Delta t}^{\mathrm{spe}}(t)$ for all the currency pairs do not independently fluctuate.

\section{Discussion}

According to [23] when calculated for systems with strong temporal correlations $\alpha$ can become time scale dependent. Specifically if Hurst exponents are inhomogeneous in terms of $\Delta t$ then the $\alpha$ values depend on the time window.

Since $\alpha$ takes values in the range from 0.8 to 0.9 at $\Delta t=1$ [min], the quotation activities of the FX market may be mutually correlated due to both internal and external factors. In order to check the dependence of the Hurst exponents on the time window we calculate 
the standard deviations $\sigma(\Delta t)$ for various window size $\Delta t$. As shown in Fig. 8 we do not confirm the Hurst exponent inhomogeneity in terms of $\Delta t$ but confirm its inhomogeneity in terms of currency pairs. On the other hand we confirm logarithmic increases of $\alpha$ in terms of $\Delta t$ for several observation weeks.

Therefore the possibility that the dependence of $\alpha$ on $\Delta t$ comes from the Hurst exponent inhomogeneity may be small. In addition to it $\alpha$ increases proportionally to $\log \Delta t$ up to $\Delta t=10$ [min]. Hence monotonically increases of $\alpha$ in terms of $\Delta t$ may be related to synchronous behavior among quotation activities. Specifically, since several market participants in the FX market conduct arbitrage operations among several currency pairs, it is thought that its synchronous behavior among currency pairs easily happens.

As shown in Fig. 3, it is found that $\alpha$ monotonically increases and has a tendency to converge into a certain value. Noting that the cut-off of a function of $\alpha$ in terms of $\Delta t$ is around $\Delta t=10$ to 20 [min]. As $\Delta t$ increases, the quotation activities may become more temporally correlated or mutually synchronous than small $\Delta t$. This implies that for a large time scale the market participants synchronously behave and may be driven by a common factor other than internal factors. Consequently, since both the temporal and mutual correlation structure among quotation activities is related to $\alpha$ 's value, the average of the correlation increases/decreases as $\alpha$ tends toward be unity/one-half. At least the average of the correlation for small $\Delta t$ seems to be smaller than that for large $\Delta t$.

These nontrivial values of $\alpha$ may imply that the market participants are affected by information both from different origins relating to microscopic dynamics and from common sources relating to macroscopic dynamics, or may imply that market participants have strong interaction. In other words the arrival rates of quotations on the brokerage systems are not independently Poissonian.

The market participants communicate via electronic broking systems and perceive both endogenous and exogenous information through electrical communication (e-mails, telephones, video-chat systems, and so on). Therefore, it is natural that they are affected by both internal and external fluctuations and/or have strong interactions with one another. When $\alpha$ descends toward $1 / 2$, the the quotations arriving on the brokerage system tends to be random. On the other hand, when $\alpha$ ascends toward 1 , the arrival tends to be synchronous. Therefore, values of $\alpha$ are useful to evaluate the states of information flows in the whole FX market.

It is known that the degree centrality is related to the degree of the component if constituents move randomly with the same probability on a network [20]. Even though the probability of the random walk is not same this assumption can be justified if multiple linkage between nodes is possible. In the FX market the relationship among market participants and currency pairs based on conveyance of quotations can be described as a bipartite network. If we can accept the assumption that quotations are randomly created by market participants then the component's centrality estimated by Eq. (5) is related to the number of market participants connected to that one.

This intuitive understanding of the FX market would be able to be mathematically written as follows. Let $N$ and $M$ denote the number of currency pairs and the number of market participants, respectively. $C_{j k}$ gives an adjacency matrix of a bipartite graph and represents a link from the $j$-th market participant to $k$-th currency pair. Then the total number of outgoing links which the $j$-th market participant has, is given by $K_{j}=$ 
$\sum_{k=1}^{N} C_{j k}$. We assume that the $j$-th market participant generates a quotation to the $k$-th currency pair with the same probability $1 / K_{j}$. Since asymmetric dynamics of quotations on a homogeneous incidence relationship can be translated into homogeneous random walk on a heterogeneous incidence relationship, we assume that the adjacency matrix can take non-negative integer values, so that $C_{j k}=0,1,2, \ldots$ Then the $k$-th currency pair's centrality may be approximated as

$$
A_{k}=\sum_{j=1}^{M} \frac{C_{j k}}{K_{j}} q_{j} /\left(\sum_{j=1}^{M} q_{j}\right),
$$

where $q_{j}$ is a probability for the $j$-th market participants to enter a quotation during $\Delta t$. Since we can assume that $q_{j}$ is a function of $t$ and the agent $j$ and the bipartite network structure of the financial markets is also time-dependent, $A_{k}$ given by Eq. (5) is also a function of time $t$. Therefore under the assumption of local stationarity we should quantify importance of currency pairs in the FX market by means of time-dependent $A_{k}$.

\section{Conclusions}

The power-law relationship between a mean of quotation activities and their standard deviation was found in the FX market. We confirmed that the scaling exponents $\alpha$ take values in a range from 0.8 to 0.9 at $\Delta t=1$ [min], and that the scaling exponents vary in time depending on observation days. We separated the common fluctuation and the specific fluctuations. It was found that the common fluctuation dominates the dynamics of hard currency pairs, while the specific fluctuations dominate that of rare currency pairs. The standard deviations of the specific fluctuations scale with $\alpha \neq 1 / 2$ as a function of means. These nontrivial values imply that the market participants may be affected by both endogenous and exogenous factors, or that they behave with a strong temporal correlation. It was found that there are groups in which currency pairs are associated with the internal/external fluctuations. Furthermore, this variation is thought to be related to synchronous and desynchronous states of information transmission in the FX market.

Observing the values of the scaling exponent $\alpha$ and the ratio between amplitude of common and specific fluctuations for different values of $\Delta t$ we found that for short time scale $\Delta t \approx 1[\mathrm{~min}]$ the dynamics of the FX market is mostly given by the internal processes while for $\Delta t$ around 1 [hour] the external factors start to be important. This observation is consistent with results of [31] on the stock market fluctuations. It means that in the stock markets and the FX market incoming news show finite time diffusion on the short time scale. On the other hand since the coherent response can be observed collective decisions occur at longer time scale.

The power-law relationship between means and standard deviations is expected to become a useful tool to quantify the total similarity among multiple time series of the FX market both massively and persistently.

As for future works, more careful analysis including relationship between auto-correlation and cross-correlation is needed. In addition to it, the persistent investigation using exhaustive data for long periods, and the mathematical modeling of the FX market from an information transmission point of view, should be conducted. One candidate for an adequate model is that of the stochastic processes $[21,16]$ and another is that of agentbased models in the financial markets $[8,29,30]$. It is inferred that the total similarity 
among behavioral parameters of agents is related to a value of the power-law exponent $\alpha$.

The understandings of the power-law relationships found in the financial markets will provide us with deeper insights on the herding behavior and economical activity via heterogeneous agents. Detailed investigations with exhaustive data sets may allow us to deeply understand them.

\section{Acknowledgments}

This work is partially supported by the Kyoto University GCOE Program "Informatics Education and Research for Knowledge-Circulating Society" (A.-H. Sato and M. Nishimura) and the Initiative for Attractive Graduate School Education (M. Nishimura) from the Japanese Ministry of Education, Culture, Sports, Science and Technology, by the EU Grant MMCOMNET, No. 012999 (A.-H. Sato and J.A. Hołyst), and by Polish Ministry of Science and Higher Education, Grants No. 1P03B04727 and 134/E365/SPB/COST/KN/DWM 105/2005-2007 (J.A. Hołyst). Furthermore A.-H. Sato is grateful for the financial support from Ken Millennium, Corporation.

\section{References}

[1] T. Mizuno, S. Kurihara, M. Takayasu, and H. Takayasu, Physica A, 324, 296-302 (2003).

[2] T. Ohnishi, T. Mizuno, K. Aihara, M. Takayasu, and H. Takayasu, Physica A, 344, 207-210 (2004).

[3] F. Petroni, and M. Serva, Physica A, 344, 194-197 (2004).

[4] Y. Aiba, and N. Hatano, Physica A, 344, 174-177 (2004).

[5] T. Suzuki, T. Ikeguchi, and M. Suzuki, Physica A, 337, 196-218 (2003).

[6] F. Wang, K. Yamasaki, S. Havlin, and H.E. Stanley, Physical Review E, 73, 026117.

[7] D. Challet and R. Stinchcombe, Physica A, 324 (2003) 141-145.

[8] A.-H. Sato, Physica A, 382 (2007) 258-270.

[9] H. Takayasu, "Advent of Econophysics", Springer-Verlag, Tokyo (2002).

[10] R. Mantegna and H.E. Stanley, An Introduction to Econophysics Correlations and Complexity in Finance (Cambridge University Press, Cambridge, 2000).

[11] M.M. Dacorogna, R. Gençay, U. Müller, R.B. Olsen, and O.V. Pictet, "An introduction to highfrequency finance", Academic Press, San Diego (2000).

[12] F. Strozzi, J.-M. Zaldìvar, J.P. Zbilut, Physica A, 312, 520-538 (2002).

[13] K. Hayashi, T. Kaizoji, and L. Pichl, Physica A, 383 (2007) 16-21.

[14] R. Albert, and A.-L. Barabási, Rev. Mod. Phys. 74 (2002) 47-97.

[15] J.A. Hołyst, J. Sienkiewicz, A. Fronczak, P. Fronczak, and K. Suchecki, Physical Review E, 72 (2005) 026108.

[16] M.A. de Menezes, A.-L. Barabási, Physical Review Letters, 92 (2004) 028701-1-028701-4.

[17] M.A. de Menezes, A.-L. Barabási, Physical Review Letters, 93 (2004) 068701-1-068701-4.

[18] Y. Kuramoto, Chemical Oscillations, Waves, and Turbulence (Springer, Berlin, 1984).

[19] A. Pikovsky, M. Rosenmlum and J. Kurths, Synchronization: A Universal Concept in Nonlinear Sciences (Cambridge University Press, Cambridge, 2003).

[20] J.D. Noh and H. Rieger, Physical Review Letters, 92 (2004) 118701-1-118701-4.

[21] A.M. Kilpatric and A.R. Ives, Nature, 422 (2003) 65-68.

[22] Z. Eisler, I. Bartos, and J. Kertész, arXiv:0708.2053v1

[23] Z. Eisler, J. Kertész, Physical Review E, 73 (2006) 046109-1-046109-7.

[24] Z.-Q. Jian, L. Guo, and W.-X. Zhou, The European Physical Journal B, 57 (2007) $347-355$.

[25] The data are provided by CQG Inc.

[26] P. Clark, Econometrica, 41 (1973) 135-155.

[27] G. Bateson, Steps to an Ecology of Mind (University of Chicago Press, Chicago, 1972). 
[28] Z. Eisler, J. Kertész, "Econophysics of Stock and other Markets", A. Chatterjee and B.K. Chakrabarti (Eds.), Springer-Verlag, Italy (2006) 49-58.

[29] S. Alfarano, T. Lux, and F. Wagner, Physica A, 370 (2006) 38-42.

[30] K. Yamada, H. Takayasu, and M. Takayasu, Physica A, 382 (2007) 340-346.

[31] Z. Eisler, J. Kertész, S.-H. Yook, and A.-L. Barabási, Europhysics Letters, 69 (2005) 664-670. 

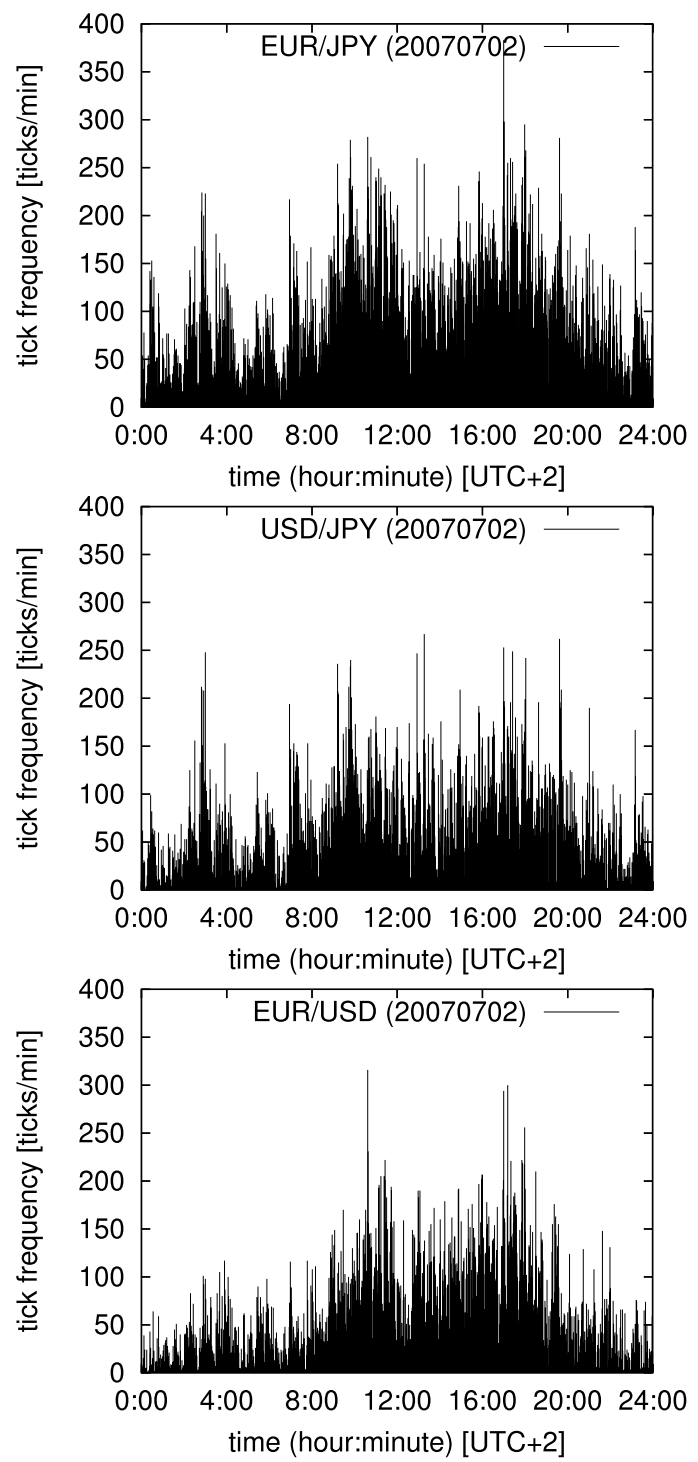

Fig. 1. Time series of quotation activities at $\Delta t=1[\mathrm{~min}]$ for EUR/JPY, USD/JPY, and EUR/USD in 2nd July 2007. 


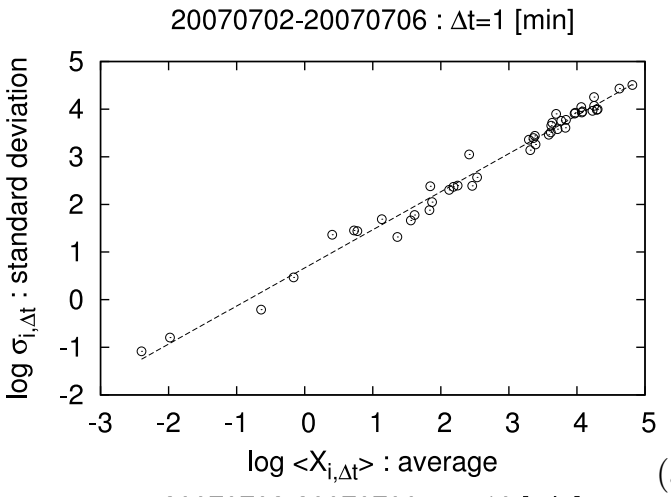

(a)

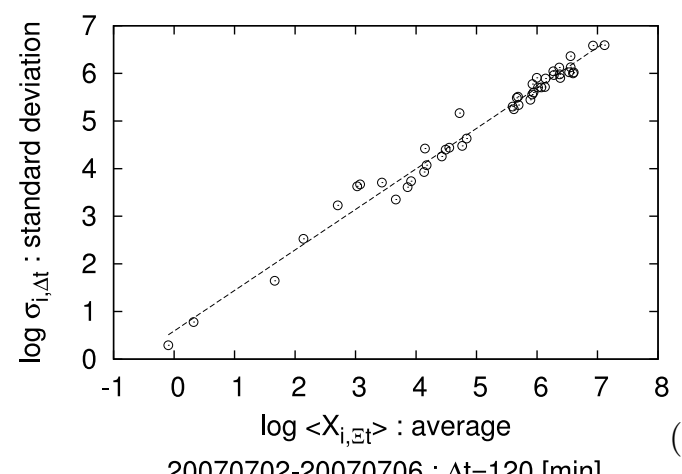

(b)

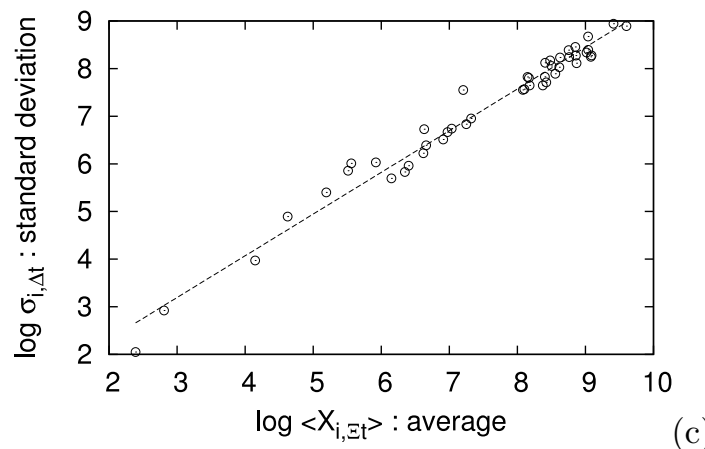

Fig. 2. Log-log plots of a mean of quotation activities and their standard deviation for each currency pair at $\Delta t=1[\mathrm{~min}](\mathrm{a}), \Delta t=10[\mathrm{~min}](\mathrm{b})$, and $\Delta t=120[\mathrm{~min}]$ (c) for a period from 2nd to 6th July 2007. The $\mathrm{x}$-axis represents a mean value, and the $\mathrm{y}$-axis a standard deviation. The straight line is estimated by using the ordinary least squares. $\log C=0.667$ and $\alpha=0.800$ at $\Delta t=1[\mathrm{~min}], \log C=0.591$ and $\alpha=0.851$ at $\Delta t=10[\mathrm{~min}]$, and $\log C=0.568$ and $\alpha=0.876$ at $\Delta t=120$ [min]. 


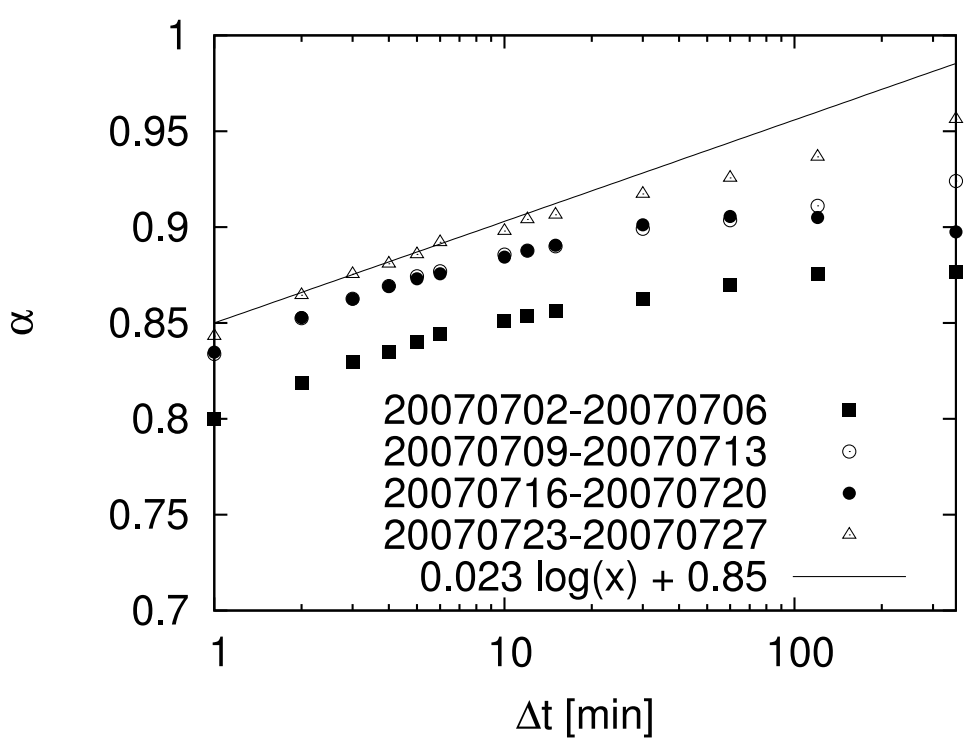

Fig. 3. A semi-log plots (bottom) of a relation between $\alpha$ and $\Delta t$ for a period from 2nd to 6th (filled square), 9th to 13th (unfilled circle), 16th to 20th (filled circle), and 23rd to and 27th (unfilled triangle) July 2007 . 


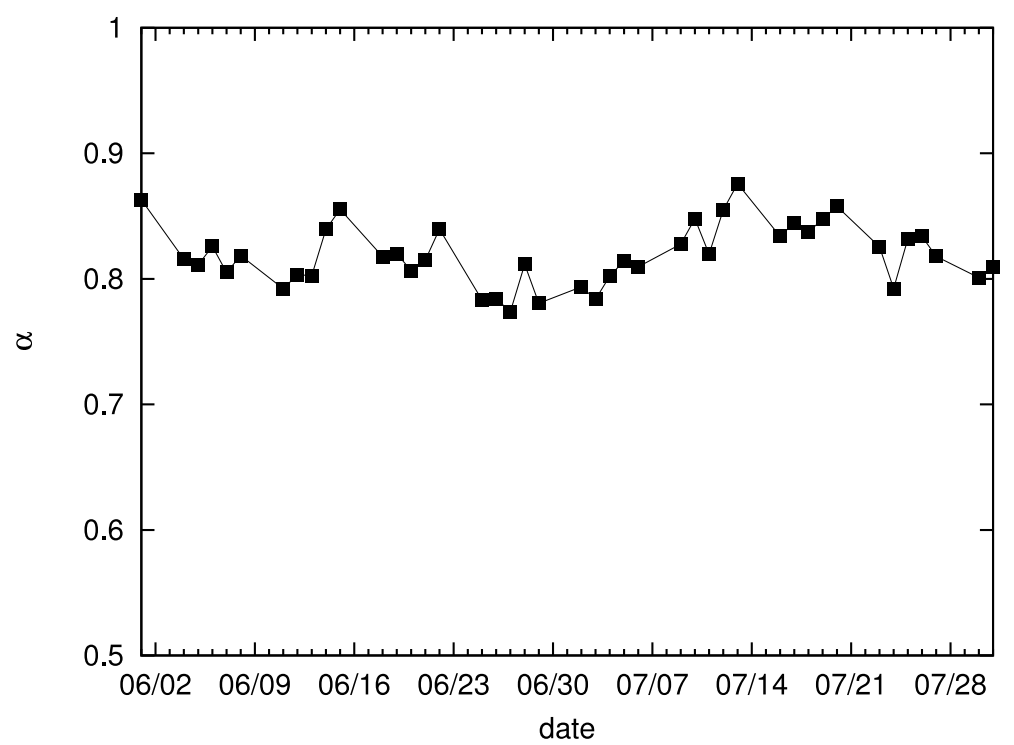

Fig. 4. Temporal variation of the scaling exponents $\alpha$ at $\Delta t=1[\mathrm{~min}]$ for periods from 1st June to 31st July 2007. 


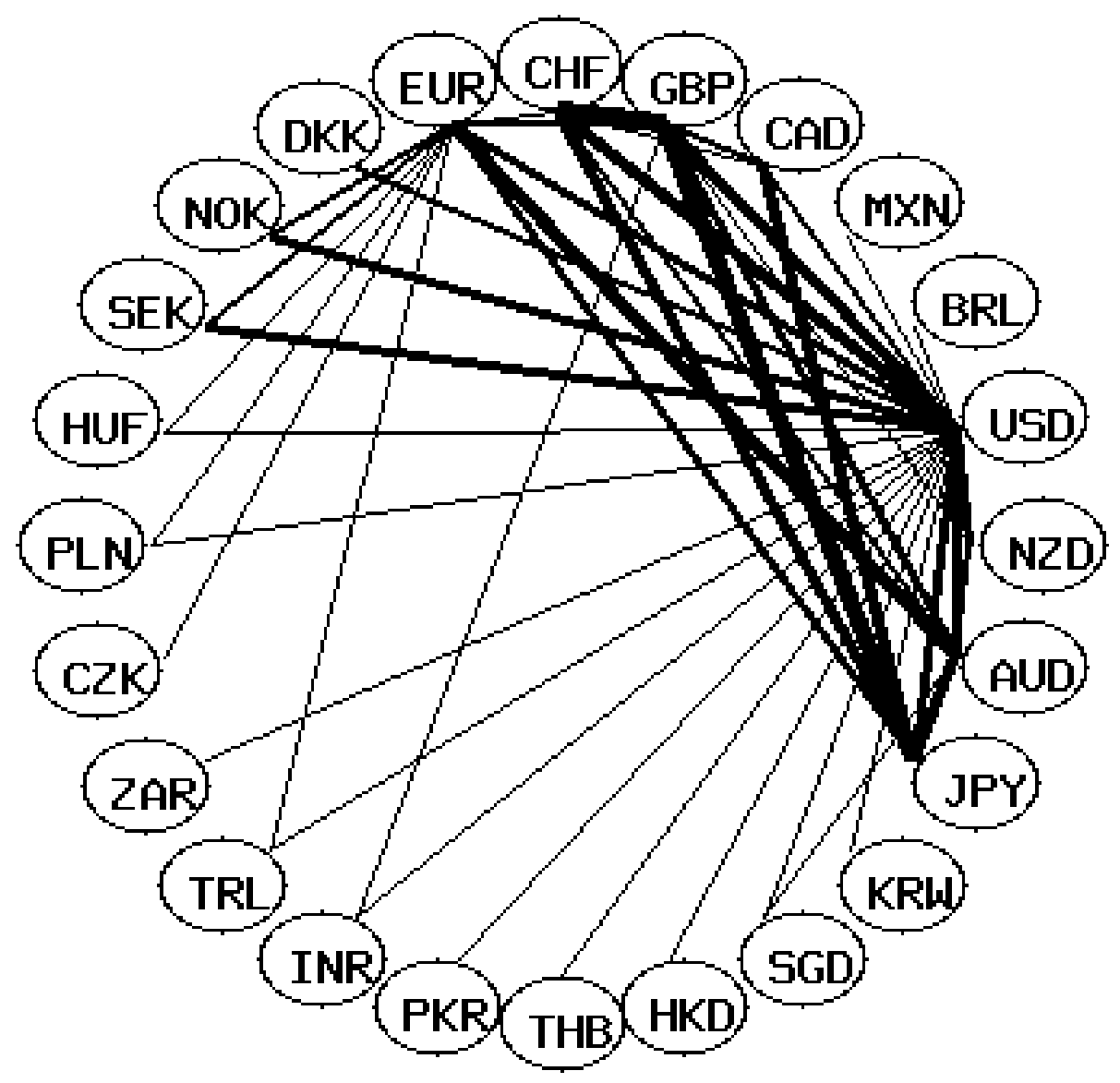

Fig. 5. Graphical illustration of 45 currency pairs in the foreign exchange market. Each node represents currency, and link between two nodes currency pairs. The link width corresponds to its centrality at 2nd July 2007. 

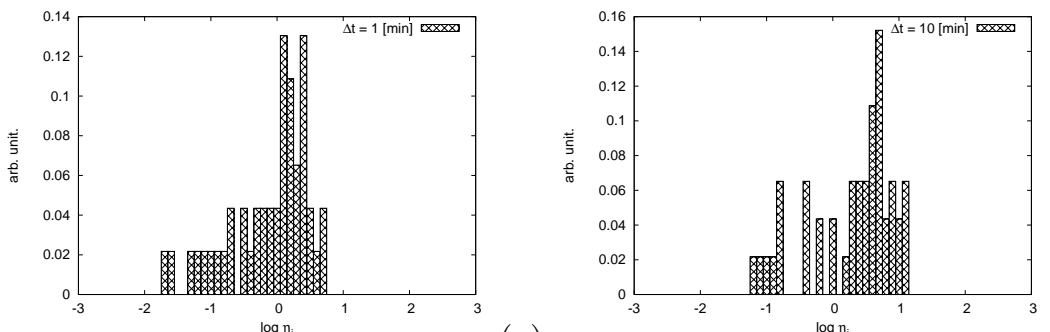

(a)

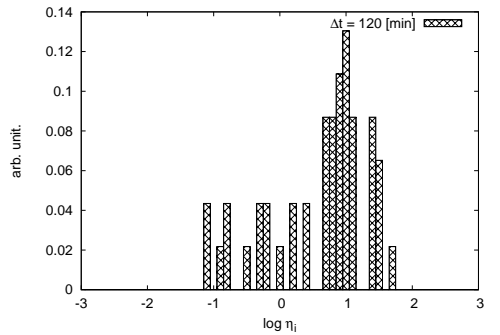

(c)

Fig. 6. Histograms of contribution of internal and external fluctuations $\left(\log \eta_{i}\right)$ at $\Delta t=1[\mathrm{~min}]$ (a mean $=-0.113$, a standard deviation $=0.627)(\mathrm{a}), \Delta t=10[\mathrm{~min}](\mathrm{a}$ mean $=0.358$, a standard deviation $=$ $0.634)(\mathrm{b})$, and $\Delta t=120[\mathrm{~min}]($ a mean $=0.807$, a standard deviation $=0.763)(\mathrm{c})$ on 2nd July 2007. 

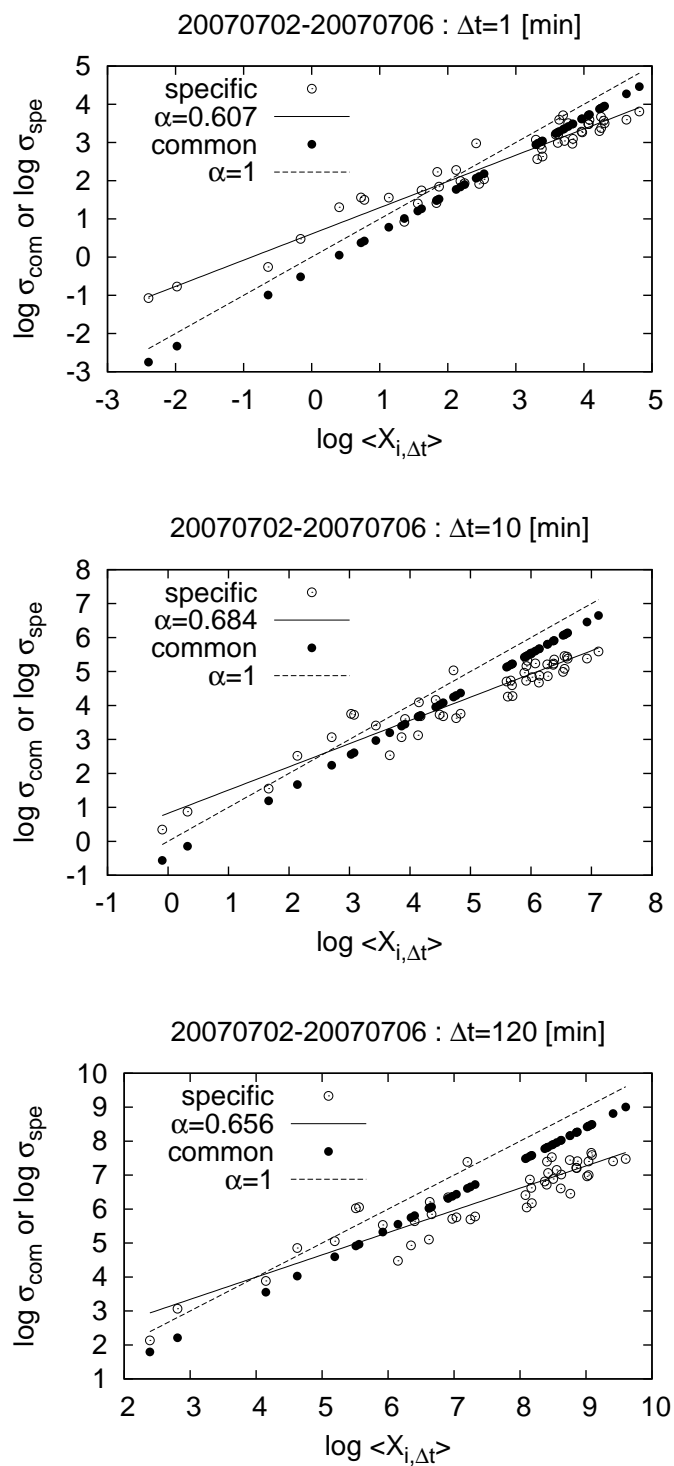

Fig. 7. Log-log plots of a mean of the specific fluctuation (unfilled circle) and of that of the common fluctuation (filled circle) and their standard deviation for each currency pair at $\Delta t=1$ [min] (a), $\Delta t=10$ [min] (b), and $\Delta t=120[\mathrm{~min}]$ (c) for a period from 2nd to 6th July 2007. The x-axis represents a mean value, and the y-axis a standard deviation. By using the ordinary least squares the straight line is estimated as $\log C=0.607$ and $\alpha=0.687$ at $\Delta t=1[\mathrm{~min}], \log C=0.825$ and $\alpha=0.684$ at $\Delta t=10$ [min], and $\log C=1.376$ and $\alpha=0.656$ at $\Delta t=120$ [min]. The slop of the dashed line is unity. 


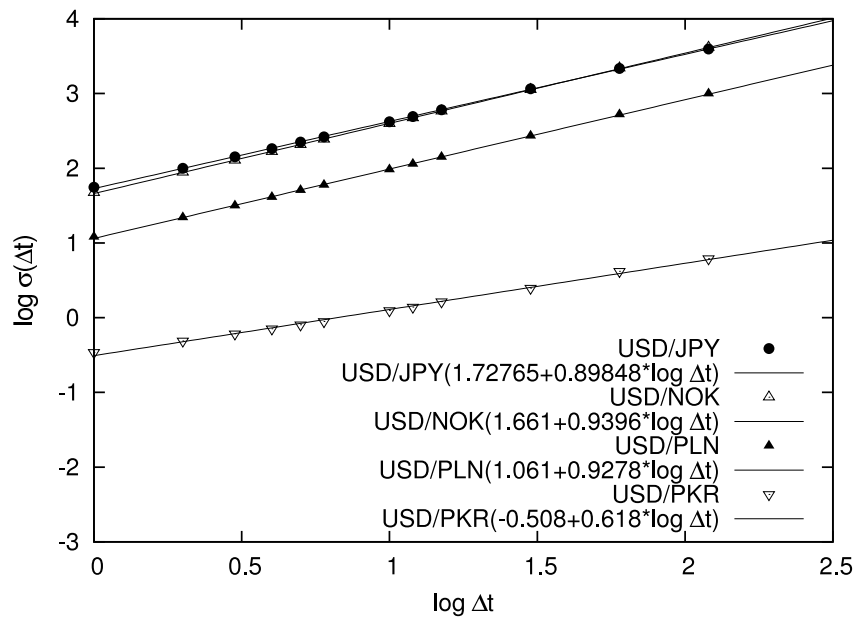

Fig. 8. A semi-log plots of a relation between standard deviation $\sigma(\Delta t)$ and $\Delta t$ of USD/JPY (unfilled circle), USD/NOK (filled circle), USD/PLN (unfilled triangle), and USD/PKR (filled triangle) for a period from 2nd to 8th July 2007. 
Table 1

The decending order of contribution of specfic and common fluctuations $\left(\log \eta_{i}\right)$ for each currency pair at $\Delta t=1[\mathrm{~min}], 10[\mathrm{~min}]$, and $120[\mathrm{~min}]$, respectively. For $\eta_{i} \gg 1$ the common fluctuation is dominant, and for $\eta_{i} \ll 1$ the specific fluctuation is dominant.



